\title{
Seiches in lateral cavities with simplified planform geometry: Oscillation modes and synchronization with the vortex shedding
}

Cite as: Phys. Fluids 32, 085103 (2020); https://doi.org/10.1063/5.0016118

Submitted: 02 June 2020 . Accepted: 16 July 2020 . Published Online: 07 August 2020

Clément Perrot-Minot, Lukas Engelen (D), Nicolas Riviere (D), Diego Lopez, Tom De Mulder, and Emmanuel Mignot (D)

\section{ARTICLES YOU MAY BE INTERESTED IN}

Investigation of the drag reduction performance of bionic flexible coating

Physics of Fluids 32, 084103 (2020); https://doi.org/10.1063/5.0016074

Analytical solutions to shock and expansion waves for non-ideal equations of state

Physics of Fluids 32, 086105 (2020); https://doi.org/10.1063/5.0015531

Effects of elasticity on unsteady forced convective heat transfer of viscoelastic fluid around a cylinder in the presence of viscous dissipation

Physics of Fluids 32, 083102 (2020); https://doi.org/10.1063/5.0009948

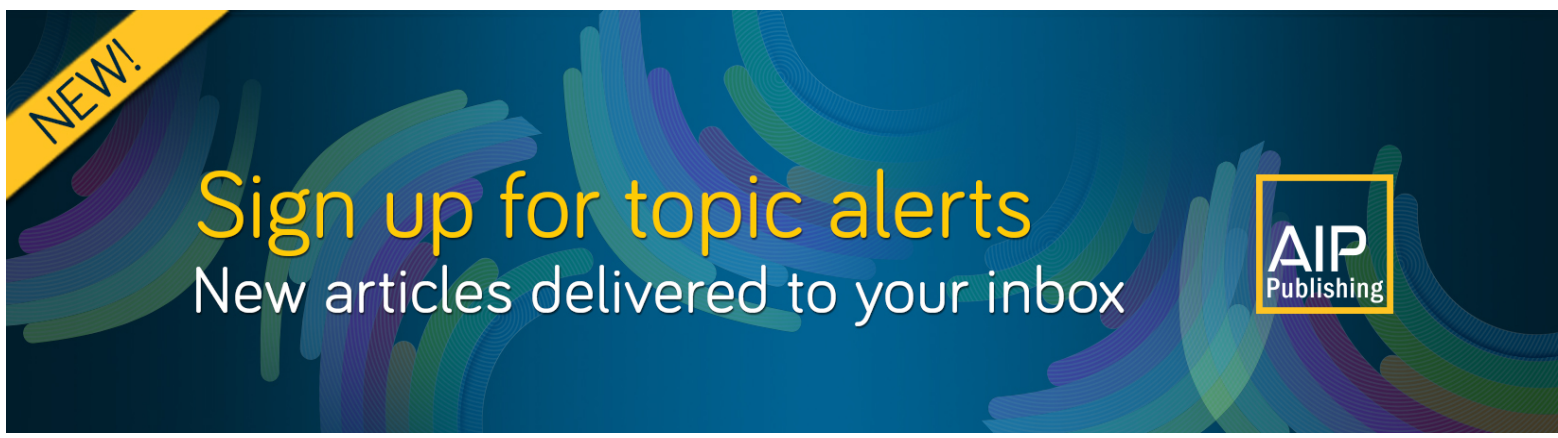




\title{
Seiches in lateral cavities with simplified planform geometry: Oscillation modes and synchronization with the vortex shedding
}

\author{
Cite as: Phys. Fluids 32, 085103 (2020); doi: 10.1063/5.0016118 \\ Submitted: 2 June 2020 - Accepted: 16 July 2020 • \\ Published Online: 7 August 2020
}

Clément Perrot-Minot, ' Lukas Engelen, ${ }^{2}$ (D) Nicolas Riviere,' (D) Diego Lopez,' Tom De Mulder, and Emmanuel Mignot ${ }^{1, a)}$ (D)

\author{
AFFILIATIONS \\ ${ }^{1}$ Univ Lyon, INSA Lyon, Ecole Centrale de Lyon, Université Claude Bernard Lyon I, CNRS, LMFA, UMR 5509, \\ 20 Avenue Albert Einstein, F-69621 Villeurbanne, France \\ ${ }^{2}$ Hydraulics Laboratory, Department of Civil Engineering, Ghent University, 9000 Ghent, Belgium
}

\begin{abstract}
a) Author to whom correspondence should be addressed: emmanuel.mignot@insa-lyon.fr
ABSTRACT

Lateral cavities adjacent to open-channel flows are dead zones located on one side of a main stream. With an approaching flow with a high (subcritical) Froude number, the free-surface of the dead-zone oscillates with high amplitudes and generates a so-called seiche. This configuration is reproduced in a rectangular cavity (with an interface length equal to the main stream channel width) in which the impact of the three dimensionless parameters (Froude number, dimensionless water depth, and geometrical aspect ratio) affecting the seiche is studied experimentally. For all configurations, a natural mode of the cavity is observed, this mode being either longitudinal or transverse, except in the case of a square cavity where bi-directional seiching occurs. Moreover, we show that while the approaching Froude number $(0.55<$ Fr $<0.7)$ and dimensionless water depth do not affect the oscillation mode, the selected natural mode is strongly dependent on the geometrical aspect ratio of the cavity. For narrow cavities (small $[W+b] / b$ with $W$ and $b$ the cavity and channel widths, respectively), a longitudinal mode occurs while for wider cavities transverse modes occur, with an increasing number of nodes as the width of the cavity increases. Finally, measuring the time-resolved 2-dimensional field of free-surface deformation in the cavity and the adjacent main stream permits us to identify the vortices shed along the mixing layer at the cavity/main stream interface and thus to analyze the synchronization between the surface oscillation and vortex shedding (at the upstream edge) and impinging (at the downstream edge) processes.
\end{abstract}

Published under license by AIP Publishing. https://doi.org/10.1063/5.0016118

\section{INTRODUCTION}

\section{A. Context}

Open-channel lateral cavity flows are dead zones located on one side of a main stream. They are typically encountered in the riverine environment. Natural lateral cavities are protected zones downstream from boulders or bank protuberances. Man-made cavities are located within groyne fields or fluvial harbors. In the coastal environment, lateral cavities can also be natural sites (typically fjords) or man-made infrastructures (harbors) connected through one side to the coastal longshore current. The natural cavities play a major role in water ecology as they constitute favorable sites for fauna and flora developments. The supply of dissolved and suspended substances to the cavity, such as nutrients, gazes, and pollutants (Sanjou et al., 2018; Sandoval et al., 2019), occurs by mass transfer across the mixing layer at the interface between the cavity and the main stream. Besides, the fluvial and coastal harbors are major infrastructures for navigation and commercial activities, for which continuity of operations is crucial for the economy.

Under specific circumstances, the water body within the cavity oscillates periodically with relatively large amplitudes. These standing waves are usually referred to as "seiches." In the coastal environment, Arneborg and Liljebladh (2001) reported large scale seiches within fjords with periods of oscillation of $20 \mathrm{~h}-40 \mathrm{~h}$ while Ardhuin et al. (2010) reported seiches within harbors with oscillation periods of $4.4 \mathrm{~min}$. When seiches occur within harbors, they may limit 

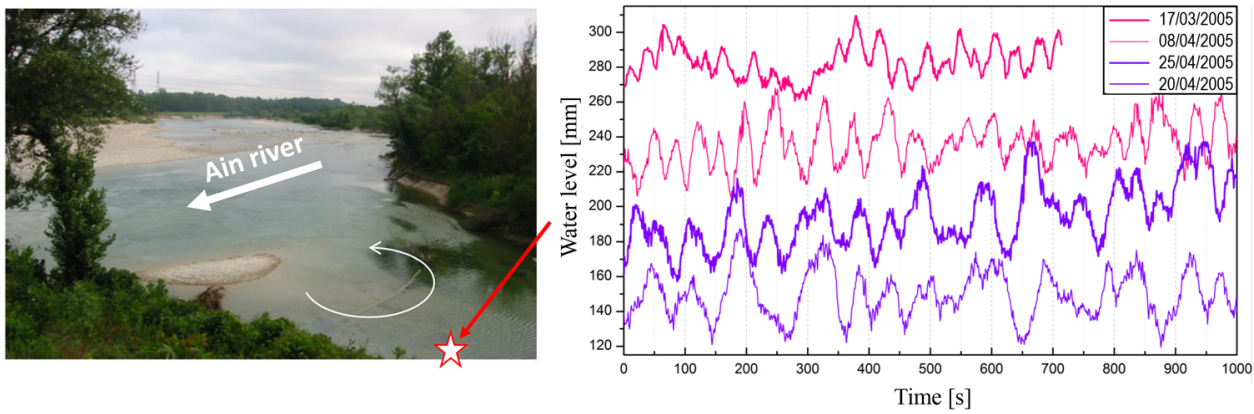

FIG. 1. Time-evolution of the water level in a natural lateral cavity in Ain River (France) registered in March and April 2005 , in the frame of the study reported by Le Coz et al. (2010) but unpublished; with courtesy of J. Le Coz.

or prevent the harbor operations for the loading and unloading of cargo on the boats. Seiches are also observed in river cavities, as measured in an oxbow by Le Coz et al. (2010) and replicated in Fig. 1, with periods of $40 \mathrm{~s}-50 \mathrm{~s}$. Seiches in river cavities play an important ecological role as they periodically aerate the plants located on the banks of the cavity and Tuna et al. (2013) suggest, based only on measured transverse velocities, an increased mass exchange coefficient between the cavity and the mean flow under seiches regime. Several causes of seiching exist, among which is the interaction of the cavity with external gravity waves (of major importance for harbors) or with a main stream with no major surface oscillation (of major importance for cavities in rivers or canals). This paper is only devoted to investigate this second cause depicted in Fig. 1.

\section{B. State of the art}

Seiches are mainly studied in laboratories under simplified geometries and hydraulic conditions with a straight, 1D, steady flow in the main stream (Kimura and Hosoda, 1997; Tsubaki and Fujita, 2006; Meile et al., 2011; Wolfinger et al., 2012; Tuna et al., 2013; Mignot et al., 2015; and Engelen et al.,2020). The cavities are usually rectangular in planform, at right angles with the main stream, with sharp edges, smooth walls, a horizontal bed, and no step between the main stream and the cavity. The peak frequencies of the free-surface oscillations measured in the rectangular cavities (Fig. 2) then fairly agree with the natural frequencies of closed rectangular basins of the same dimensions: $L \times(W+b) \times h$ (see Fig. 2 ), with $L$ and $W$ the

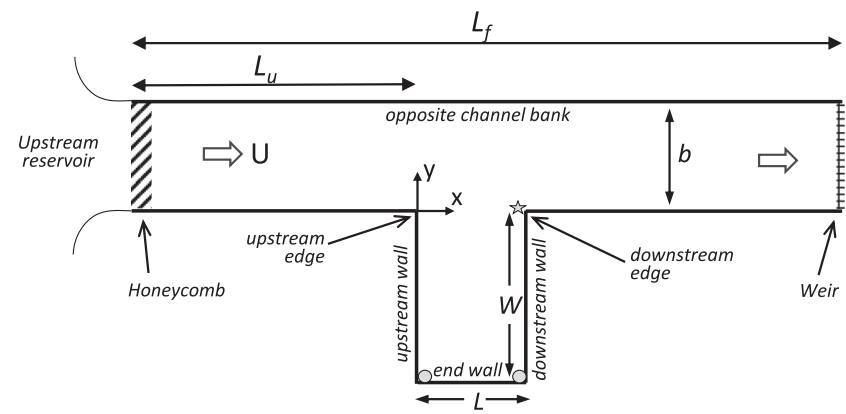

FIG. 2. Sketch of the experimental setup with the gray star and circles indicating the location of ultrasonic (at Lyon and Ghent laboratories) and pressure (at Ghent laboratory) sensors. length and width of the cavity, respectively, along $x$ and $y$ axes, $b$ the channel width, and $h$ the mean water elevation. Natural frequencies $f$ of closed rectangular basins were studied (among others) by Lamb (1945) and Rabinovich (2009), and read as follows:

$$
\left\{\begin{aligned}
f_{n_{x} n_{y}} & =\frac{c}{2}\left[\left(\frac{n_{x}}{L}\right)^{2}+\left(\frac{n_{y}}{W+b}\right)^{2}\right]^{\frac{1}{2}}, \\
c & =\frac{g}{2 \pi f_{n_{x} n_{y}}} \tanh \frac{2 \pi h f_{n_{x} n_{y}}}{c},
\end{aligned}\right.
$$

where $n_{x}$ and $n_{y}$ are the numbers of nodes along, respectively, the $x$ and $y$ direction, $c$ is the celerity of the water waves, and $g$ is the acceleration of gravity. Following Rabinovich (2009), in this study, Fig. 3 shows a selection among the simplest one-dimensional natural modes $m_{n_{x} n_{y}}$ of rectangular cavities oscillating at the corresponding natural frequency $f_{n_{x} n_{y}}$ predicted by Eq. (1). These 1D modes exhibit antinodes along opposite walls and can exhibit one or several additional antinode and node lines in between. For longitudinal modes, the oscillation is confined between the upstream and downstream wall of the cavity (Fig. 2) and no major oscillation is observed within the main stream. Oppositely, for transverse modes, the oscillation occupies the whole available width from the opposite bank of the channel up to the end wall of the cavity (Fig. 2) such that the water surface periodically oscillates in both the cavity and the channel.

Several authors investigated the frequencies and amplitudes of the free-surface oscillations in single lateral cavities adjacent to a main stream. For a given cavity geometry, two different flow regimes were reported in the literature as a function of the main stream Froude number $F r=U / \sqrt{g h}$, with $U$ the main stream bulk velocity.

First one is for low Froude numbers $(\mathrm{Fr}<0.6)$. Wolfinger et al. (2012) observed a linear increase of the oscillation frequency with Fr. Perrot-Minot et al. (2020) explained this behavior, showing that the frequency is a solution of the Rossiter (1964) feedback model, adapted to free-surface flows. However, Perrot-Minot et al. (2020) reported that, as Fr increases, the free-surface oscillation frequency evolves along consecutive plateaus instead of a linear increase, the frequency of each plateau being equal simultaneously to (i) a natural frequency of the cavity and, thus, a solution of Eq. (1) and (ii) a solution of the adapted Rossiter (1964) model. Nevertheless, all authors agree that the surface oscillation remains of limited amplitude in this low Froude number regime.

The second regime is for higher Froude numbers $(\mathrm{Fr}>0.6)$. Different authors observed surface oscillations with a much higher 


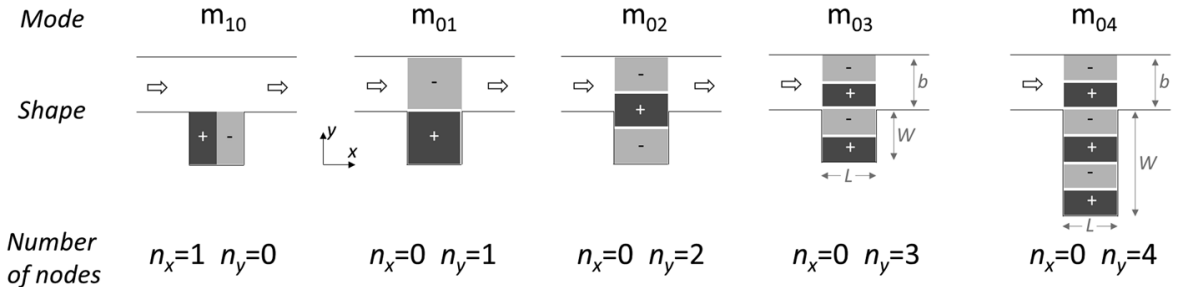

FIG. 3. Sketch of five among the simplest 1-dimensional natural oscillation modes, where + and - zones correspond to a superelevation and underelevation of the free-surface compared to the undisturbed surface condition. amplitude than for the first regime. Tsubaki and Fujita (2006) reported amplitudes of the free-surface oscillation up to $15 \%$ of the mean water depth. Besides Wolfinger et al. (2012), Tuna et al. (2013) and Engelen et al. (2020) reported an increasing amplitude up to Fr $\sim 0.7$ followed by a decreasing amplitude for higher Froude numbers. Regarding the oscillation modes, Kimura and Hosoda (1997) (for calculations with an aspect ratio of $[W+b] / b=2.5$ ), Wolfinger et al. (2012) and Tuna et al. (2013) (for measurements with $[W+b] / b=2$ ), and Perrot-Minot et al. (2020) (for measurements with $[W+b] / b=2.66)$ reported peak frequencies equal to the natural frequency of the first longitudinal cavity mode $\mathrm{m}_{10}$ [Eq. (1)] with a single node along the $x$ axis $\left(n_{x}=1\right)$ and none along $y\left(n_{y}\right.$ $=0$ in Fig. 3). Besides, Mignot et al. (2015) and Engelen et al. (2020) studied the specific case of a cavity where the transverse extension of the cavity plus the main stream equals twice the cavity length: $W+b=2 L$. In this specific geometry, the authors observed a socalled bi-directional seiche composed of two simultaneous modes, a longitudinal $\mathrm{m}_{10}$ and a transverse $\mathrm{m}_{01}$ mode, with a temporal alternation of domination by one of the modes, in terms of wave amplitude.

Kimura and Hosoda (1997), Wolfinger et al. (2012), and Perrot-Minot et al. (2020) observed that the period of the seiche equals that of the large-scale vortices generated at the upstream edge and traveling along the mixing layer at the interface between the cavity and the main stream toward the downstream edge (Mignot et al., 2016). Moreover, Tuna et al. (2013) measured increasing turbulent kinetic energy and Reynolds stresses as the amplitude of the seiches increases. These results confirm the strong interaction between the turbulence dynamics in the mixing layer and the oscillation of the free-surface.

\section{Strategy and objectives}

The present work is dedicated to the oscillations of the freesurface in the high Froude number regime $(\mathrm{Fr}>0.6)$ and their interactions with the vortex shedding process along the mixing layer. The literature review reveals that no consensus is available regarding the selection of the oscillation mode for a given flow configuration. The first objective is to identify how the geometrical and flow dimensionless parameters govern the selection of the seiching modes. Moreover, while a consensus seems to emerge that the vortex impingement at the downstream edge of the cavity is responsible for the seiching process, the coupling between both processes was never measured. The second objective of the present work is to characterize the synchronization between the vortex dynamics and the oscillation modes.

Section II introduces the experimental setup, measurement techniques, and the dimensional analysis governing the seiche phenomenon. Section III presents the impact of the different dimensionless parameters on the oscillation modes. Section IV concerns the synchronization of the surface oscillation with the vortex shedding process. Finally, the results are discussed and conclusions are given in Sec. $V$.

\section{EXPERIMENTAL APPROACH}

\section{A. Experimental setup}

The experiments presented in this paper were performed in two laboratories: (i) the Fluid Mechanics and Acoustics Laboratory (LMFA) of the Universite de Lyon and (ii) the Hydraulics Laboratory of Ghent University. Both facilities are very similar to each other. Each one consists of a horizontal, straight, smooth flume of width $b$ and total length $L_{f}$ connected, at a distance $L_{u}$ from the entrance, to an adjacent horizontal smooth rectangular cavity of length $L$ and width $W$, with no step at the connection between both (Fig. 2). The inlet discharge $Q$ is adjusted and measured in the pumping loop using an electromagnetic flowmeter (from Endress Hauser with an accuracy of $0.005 \mathrm{l} / \mathrm{s}$ at Lyon and MagMaster from ABB with an accuracy of $0.003 \mathrm{l} / \mathrm{s}$ at Ghent). From the upstream reservoir to the channel, the water passes through a $3 \mathrm{D}$ vertical and transverse convergent to homogenize the velocity distribution across the channel section. Moreover, to straighten the inflow, a honeycomb is installed at the inlet of the convergent nozzle followed by grids at the outlet of the nozzle, i.e., at the entrance of the channel. Figure 16 in Appendix A plots transverse profiles of the approaching mean streamwise velocity and turbulent intensity for a selected configuration. At the outlet, the water flows above a thin adjustable weir that permits us to adjust the mean water depth $h$ in the channel in front of the cavity. The dimensions of the facilities are listed in Table I. Note that both experimental setups correspond to the specific geometrical configuration case with $b / L=1$.

\section{B. Measurement methods}

Both flumes are equipped with an ultrasound sensor located close to the downstream edge of the cavity (Fig. 2) that records at

TABLE I. Characteristics of the facilities at Ghent laboratory and Lyon laboratory along with the range of Reynolds $\mathrm{Re}_{\mathrm{Dh}}=\mathrm{UD}_{\mathrm{h}} / v$ with $\mathrm{D}_{\mathrm{h}}$ the hydraulic diameter in the main stream and Froude $\mathrm{Fr}=\mathrm{U} /(\mathrm{gh})^{0.5}$.

\begin{tabular}{lccccccc}
\hline \hline & $b(\mathrm{~m})$ & $L_{u}(\mathrm{~m})$ & $L_{f}(\mathrm{~m})$ & $L(\mathrm{~m})$ & $W(\mathrm{~m})$ & $R e_{\mathrm{Dh}}$ & $\mathrm{Fr}$ \\
\hline Lyon & 0.3 & 2 & 4.9 & 0.3 & $0.1-2$ & $0.7-0.8 \times 10^{5}$ & $0.55-0.65$ \\
Ghent & 0.4 & 3 & 12 & 0.4 & 0.4 & $2.2-2.7 \times 10^{5}$ & $0.55-0.68$ \\
\hline
\end{tabular}



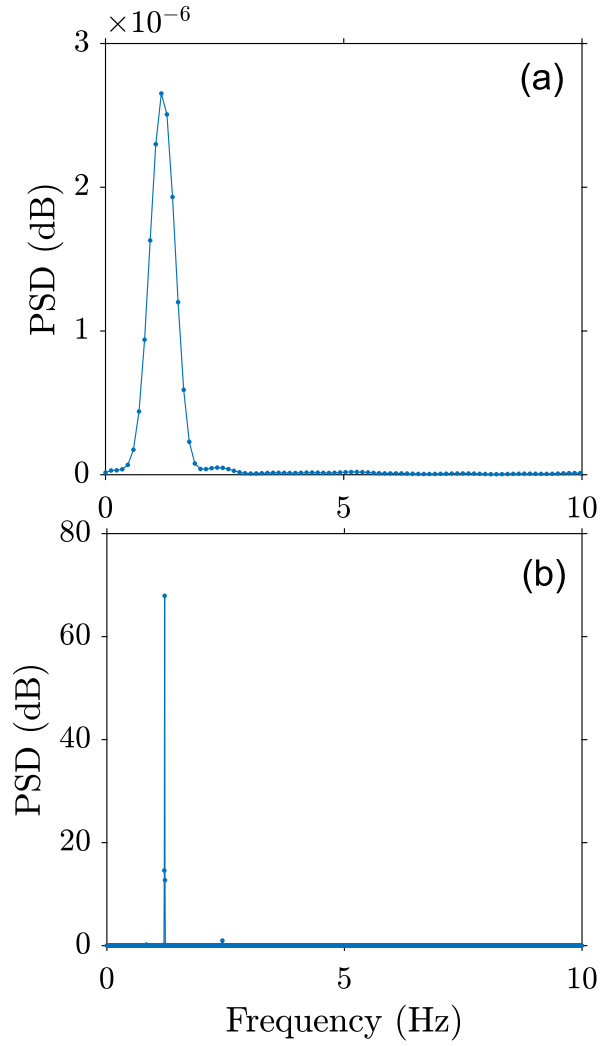

FIG. 4. Comparison of the spectra of the water depth registration by the profilometry (a) and ultrasound (b) methods near the downstream edge of the cavity for the same configuration with $\mathrm{Fr}=0.6$ and $[\mathrm{W}+\mathrm{b}] / \mathrm{b}=2.66$ in series $2 \mathrm{~b}$.

high frequency the temporal evolution of the water depth with a water depth resolution $d h=0.3 \mathrm{~mm}$. A UNDK $2016912 \mathrm{~S} 35 \mathrm{~A}$ of Baumer Electrics is used at Lyon with a sampling frequency of $30 \mathrm{~Hz}$ with measurement durations of $10 \mathrm{~min}$ and at Ghent with a sampling frequency of $50 \mathrm{~Hz}$ with measurement durations of $90 \mathrm{~min}$. Spectra of the recorded signals then permit us to identify the peak frequency of the free-surface oscillation near the downstream edge of the cavity [Fig. 4(b)].
However, the ultrasound sensors only permit us to assess the oscillation of the free-surface at a single location. Therefore, both flumes are equipped with additional measurement devices to gain information on the shape of the free-surface oscillation. In the flume in Ghent, two pressure sensors (UNIK5000 of GE Measurement and Control) simultaneously register the time evolution of the water depth at the two inner corners of the cavity end wall (Fig. 2) at a sampling frequency of $50 \mathrm{~Hz}$, with a measurement accuracy of $\pm 0.05 \mathrm{~mm}$. The pressure sensors are synchronized with the ultrasound sensor. The flume in Lyon is additionally equipped with a fringe projection profilometry method, similar to that described by Cobelli et al. (2009) and Aubourg and Mordant (2016). This method consists of projecting a pattern composed of sinusoidal fringes on the free-surface via a video-projector (Epson EH-TW6800, resolution $1920 \times 1080$ pixels, lighting power 2700 lumens) located about $2 \mathrm{~m}$ above the flume and projecting downward. The water is made opaque using demineralized water mixed with a concentration of $20 \mathrm{~g} / \mathrm{l}$ of titane dioxide to better reflect the projected pattern. Using titane dioxide instead of dye maintains the same surface tension and viscosity as plain water, while the use of demineralized water solves the problem of an inefficient colloidal mixing of titane dioxide in tap water. A video-camera with a high spatial resolution (2048 $\times 1544$ pixels) and high optical sensibility is installed just next to the projector, which records the projected pattern from above at 30 frames per second. Two linear polarizers shifted by $90^{\circ}$ are used, one being installed on the projector and the second on the camera. This strongly reduces the direct reflection of light projected by the video-projector on the water surface and reflected toward the camera. As the free-surface gets deformed, due to, for example, an oscillation mode, the fringes also deform and the camera records a modified image of the fringe pattern. Based on the optical rules described by Cobelli et al. (2009), the algorithm computes the difference of elevation between the reference pattern (recorded on a flat water surface of same mean elevation) and the deformed pattern, corresponding to the surface oscillation.

Repeating this process for each pixel in each recorded image finally permits us to reconstruct the $2 \mathrm{D}$ deformation of the freesurface at high spatial $(0.4 \mathrm{~mm}$ over the whole cavity and channel width) and temporal (every 1/30 s) resolution. To test the uncertainties in vertical elevation and horizontal location, a pyramid was created with a $3 \mathrm{D}$ printer and installed within the cavity (without water). Figure 5 compares the exact shape of the pyramid with the

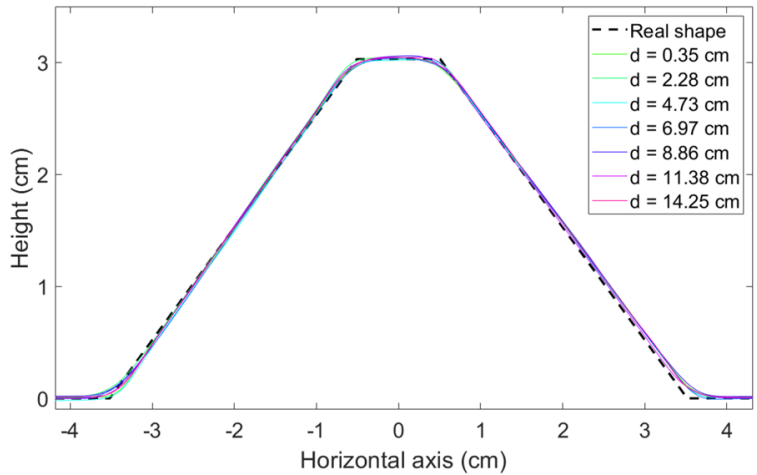

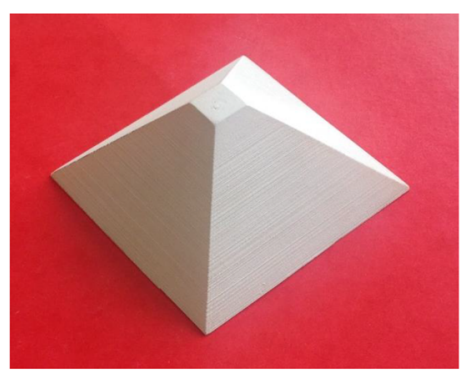

FIG. 5. Validation of the fringe projection profilometry method by comparing the geometry of a 3D-printed pyramid (right) with the corresponding shape measurement (left) as the pyramid is moved away at distance $d$ from the center of the domain. 
shape measured by profilometry at increasing horizontal distances from the center of the projected pattern. It reveals that the maximum difference in elevation and horizontal location of the reconstructed pyramid is limited to $400 \mu \mathrm{m}$, which corresponds to the pixel size [as for Cobelli et al. (2009)]. Figure 4 compares the amplitudes and spectra of the free-surface elevations measured near the downstream edge for a selected flow configuration with the ultrasound and profilometry method. Although the profilometry method increases the width of the corresponding spectral peak due to measurement durations limited to about $20 \mathrm{~s}$ (due to the high quantity of data to be stored at high frequency), both spectra show the same trend with a difference in peak frequency limited to $0.05 \mathrm{~Hz}$ and a difference in measured oscillation amplitudes of $6.6 \%$. These verifications prove the quality of this surface elevation measuring method. Although the technique requires $1.2 \mathrm{~m}^{3}$ of demineralized water in present experiments, it enables to reconstruct the surface deformation with high spatial and time resolutions.

For oscillations with periodic deformation of the free-surface in the cavity, which exhibit a distinct peak in the recorded spectrum (Fig. 4), the computed deformation can be projected onto the corresponding Fourier mode in order to filter out the associated noise and better identify the shape of the oscillation. In particular, the frequency of the selected Fourier mode is taken equal to the peak frequency and for each pixel, the magnitude of the free-surface deformation is then taken equal to the root-mean-square value of the raw data for this pixel, multiplied by the phase of oscillation along the reconstructed period. Figure 6 compares snapshots (spatial distribution of surface deformation $\eta$ made non-dimensional by the mean water depth $h$, with $\eta=z_{w}-h$ and $z_{w}$ the instantaneous local surface elevation) at a given time before (raw data) and after projection on the Fourier mode of the peak frequency, in which the latter appears less noisy and facilitates identifying the excited seiching mode.

It is common knowledge that free-surface oscillations take much time to establish to a steady state. Figure 7 shows the (a)

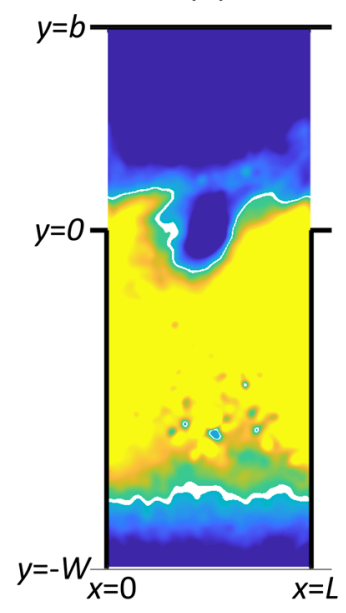

(b)

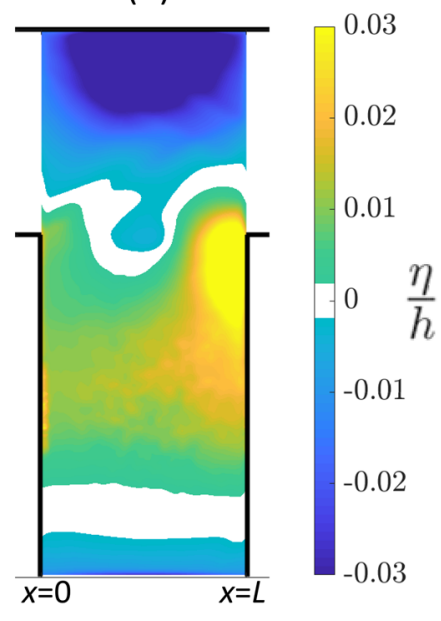

FIG. 6 . Snapshot at $t / T=0.5$ of free-surface deformation by fringe projection: comparison between raw data (a) and the projected solution on the Fourier mode $\mathrm{m}_{02}$ of peak frequency $\mathrm{f}_{02}(\mathrm{~b})$ for $[\mathrm{W}+\mathrm{b}] / \mathrm{b}=3.33$ and $\mathrm{Fr}=0.6$ in series 3 .
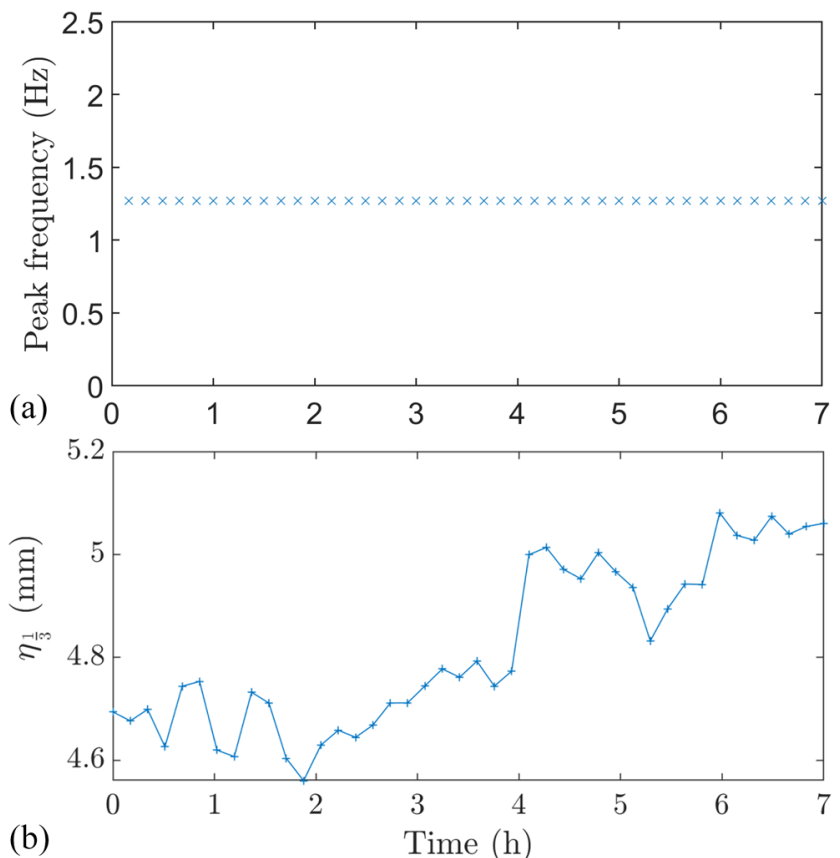

FIG. 7. Time evolution of the peak frequency (a) and amplitude (b) of the free-surface oscillation measured at the downstream edge of the cavity by the ultrasound sensor for flow with $[\mathrm{W}+\mathrm{b}] / \mathrm{b}=2.66$ and $\mathrm{Fr}=0.62$ in series $2 \mathrm{~b}$.

evolution along several hours of the peak frequency of the ultrasound sensor signal spectrum and of the oscillation amplitude $\eta_{1 / 3}$. The latter is defined as the arithmetic average of the highest one third half crest-to-trough local wave amplitude. The time required to reach a steady state strongly differs between the two parameters. The natural mode and associated natural frequency are very rapidly reached and do not change with time any further. Oppositely, while the order of magnitude of the oscillation amplitude is reached after only $10 \mathrm{~min}$, several hours are required to reach a quite established seiche amplitude [this is not true for bi-directional seiching, where the surface oscillation switches from one dominant mode to another over long periods, as discussed in detail by Engelen et al. (2020)].

Finally, to verify the repeatability of the measured surface deformation by profilometry, the same flow configuration is reproduced 9 times at different days for which the variability of measured peak frequency remains below 1\% (see Fig. 17 in Appendix B).

\section{Measurement procedure}

The procedure applied to measure the surface oscillation aims at ensuring an independent measurement for each configuration from the previous ones, i.e., preventing from any hysteresis phenomenon. This procedure is as follows: (1) The inflow discharge and weir crest height are first adjusted to the desired values. (2) The cavity is closed with a thin plate inserted along the interface $(y=0)$ to damp the oscillation within the cavity. (3) The plate is then gently removed to connect the cavity to the main stream. (4) Once a steady-state is reached, both in terms of oscillation frequency and amplitude, measurements are performed using the ultrasound and 
TABLE II. Experimental configurations. Detailed configurations along with measured peak frequencies are listed in Appendix C. Boldface text refers to the modified dimensionless parameter.

\begin{tabular}{lcccccc}
\hline \hline & $F r$ & $h / L$ & {$[W+b] / b$} & $b / L$ & Performed at & Figures \\
\hline Series 1 & 0.6 & $\mathbf{0 . 2 5 - 0 . 3 8}$ & 2 & 1 & Ghent laboratory & None \\
\hline Series 2a & $\mathbf{0 . 5 5 - 0 . 6 8}$ & 0.36 & 2 & 1 & Ghent laboratory & None \\
Series 2b & $\mathbf{0 . 5 5 - 0 . 6 5}$ & 0.195 & 2.66 & 1 & Lyon laboratory & Fig. 9, Appendix A \\
Series 2c & $\mathbf{0 . 6 - 0 . 6 5}$ & 0.195 & 3 & 1 & Lyon laboratory & Fig. 9 \\
\hline Series 3 & 0.6 & 0.195 & $\mathbf{1 . 3 3 - 7 . 6 6}$ & 1 & Lyon laboratory Figs. 10-15, Appendixes A and B \\
\hline \hline
\end{tabular}

pressure sensors and, for most configurations in Lyon, the profilometry method. The peak frequency is obtained from the spectrum of the ultrasound recordings [Fig. 4(b)]. Note that the absence of peak frequency in the spectrum of velocity signals measured in the upstream branch of the main stream (not shown here) permitted to verify that the possible vibrations of the facility play no role in the selection of the oscillation mode.

Besides, at Lyon, the oscillation mode is identified by looking directly at the flume and on the reconstructed free-surface oscillations based on the profilometry data, when available (as depicted in Sec. IV). At Ghent, the analysis of the synchronized signals from the ultrasound and both pressure sensors permits us to distinguish the oscillation mode by evaluating the cross-spectra between the three recorded time series (Engelen et al., 2020).

\section{Dimensional analysis and studied configurations}

For a rectangular cavity with smooth vertical walls adjacent to a main stream in highly turbulent regime, the dimensional analysis ruling the selected mode $m$ and peak frequency $f$ of the free-surface oscillation is similar to those proposed by (among others) Jackson et al. (2015) and Mignot et al. (2017) and reads (discarding the effect of the viscosity and wall roughness, that would introduce, respectively, a Reynolds number and a dimensionless roughness or friction coefficient) as follows:

$$
(m, f)=\Phi(\mathrm{Fr}, h / L, b / L,[W+b] / b) .
$$

Note that in the present work, $b / L=1$, so that Eq. (2) reduces to

$$
(m, f)=\Phi(\mathrm{Fr}, h / L,[W+b] / b),
$$

where $[W+b] / b$ could be replaced by $W / L$ and $h / L$ could be replaced by $h / b$ in the dimensional analysis (due to $b / L=1$ ). The aim of Sec. III is to determine the influence of each of the three nondimensional parameters on the oscillation mode and peak frequency of the cavity. For that purpose, three measurement series are considered (Table II) where either the water depth alone, the Froude number alone or the geometrical aspect ratio of the cavity alone is varied in, respectively, series $1-3$. Note that series 2 with the varying Froude number is repeated three times for different values of the two other parameters.

\section{RESULTS: OSCILLATION MODES, OSCILLATION AMPLITUDE, AND PEAK FREQUENCIES}

Figure 8 reveals that the free-surface oscillation measured by profilometry exhibits the same peak frequency all over the cavity and the main stream, and equals the peak frequency $f$ measured by the ultrasound sensor at the downstream edge (Fig. 4). This result is verified on all flow configurations. For the longitudinal mode shown in Fig. 8, the maximum oscillation amplitude is observed at the downstream edge (at point 1 ) and within the cavity near the upstream and downstream walls (points 4 and 5). Oppositely, the amplitudes at the upstream edge (point 2), in the main stream (points 3 and 7), and near the node line (point 6) remain much lower.

The impact of each of the three non-dimensional parameters from Eq. (2) on the free-surface oscillation mode in the cavity and associated peak frequency is assessed in the next three sub-sections.

\section{A. Influence of the non-dimensional water depth}

Tsubaki and Fujita (2006) suggested that the normalized water depth $h / L$ does not affect the selected oscillation mode and

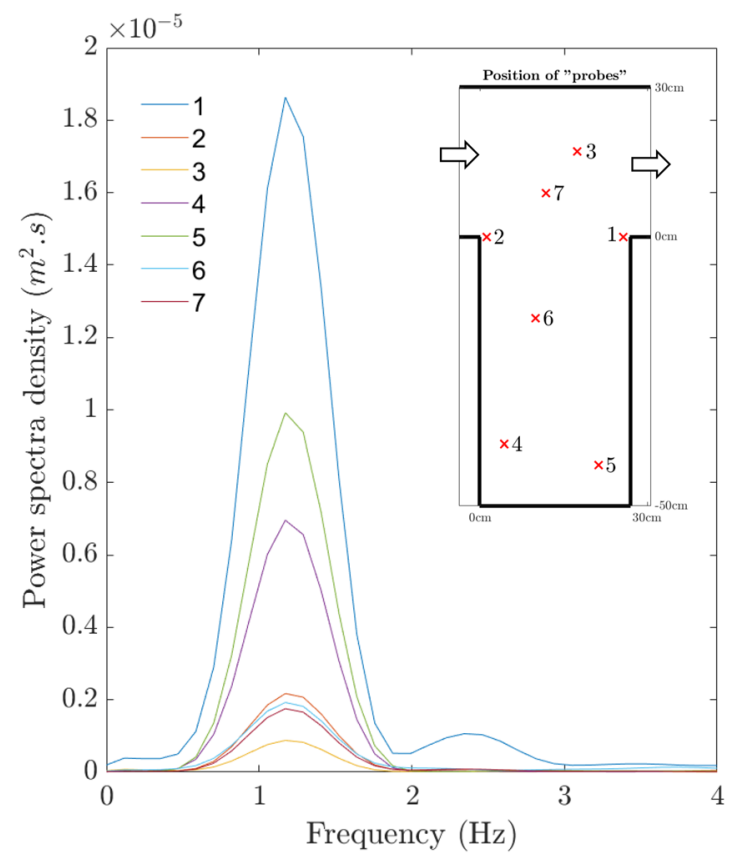

FIG. 8. Comparison of the spectra of water depth signals measured in different locations of the cavity and main stream for a configuration with a longitudinal mode $m_{10}$ 
corresponding frequency for $h / L$ from 0.08 to 0.2 . This result is confirmed by Engelen et al. (2020) for series 1 (Table II) for $h / L$ $=0.25-0.38$ in a square cavity with bi-directional seiching where both $m_{10}\left(f=f_{10}\right)$ and $m_{01}\left(f=f_{01}\right)$ co-exist for all tested configurations. Moreover, the authors showed that while the amplitude of oscillation changes significantly with $h / L$, especially for the longitudinal mode $m_{10}\left(f=f_{10}\right)$, no clear relationship between oscillation amplitude and normalized water depth $h / L$ could be sorted.

\section{B. Influence of the Froude number}

For a cavity of aspect ratio equal to $[W+b] / b=2$, Wolfinger et al. (2012) and Tuna et al. (2013) reported that the oscillation mode remains equal to $m_{10}\left(f=f_{10}\right)$ for a Froude number Fr increasing from 0.55 to 0.85 . This locking behavior is confirmed by all three series 2 (Table II). For series $2 \mathrm{a}$ in a square cavity, the same bidirectional seiching as in series 1 occurs for Fr from 0.55 to 0.68 . For series $2 \mathrm{~b}$ in a relatively narrow cavity, the oscillation mode $\mathrm{m}_{10}$ ( $\left.f=f_{10}\right)$ occurs for Fr from 0.55 to 0.65 , as already discussed by Perrot-Minot et al. (2020). For series $2 \mathrm{c}$ in a wider cavity, the transverse oscillation mode $\mathrm{m}_{02}\left(f=f_{02}\right)$, with two nodes across the main stream and the cavity, occurs for Fr from 0.6 to 0.65 . All these results from the literature and present work prove that the Froude number

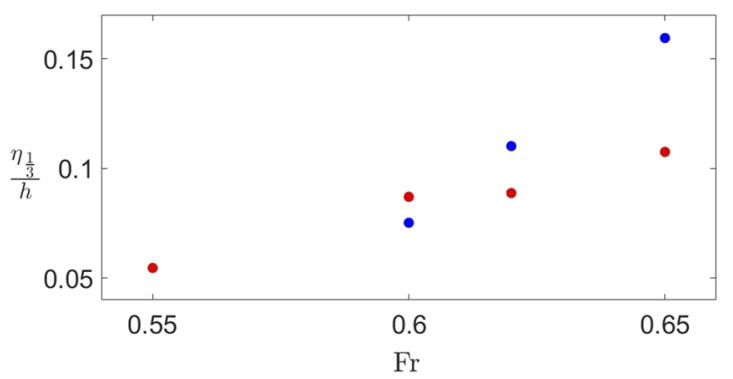

FIG. 9. Evolution of the normalized oscillation amplitude with the Froude number for series $2 \mathrm{~b}$ (red) and $2 \mathrm{c}$ (blue). The authors wish to remind that $\eta_{1 / 3}$ quantifies the amplitude of surface oscillation at the downstream edge of the cavity, which is not necessarily an antinode of the oscillation. The amplitude of the standing wave should be systematically measured at the antinode.

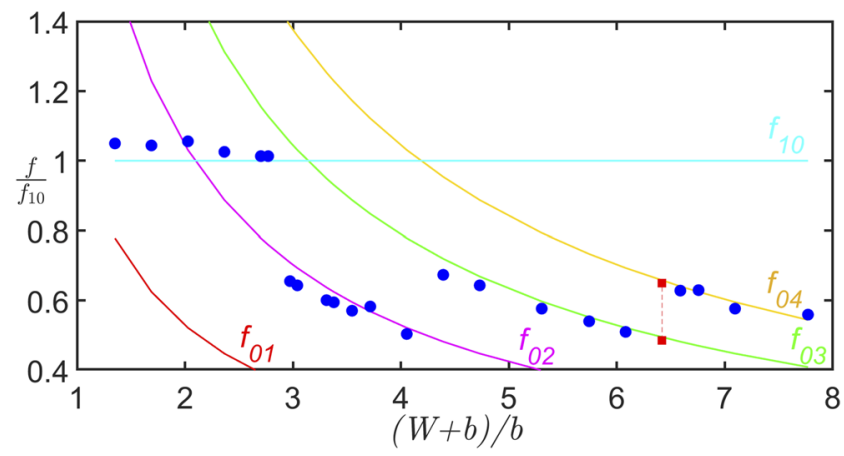

FIG. 10. Influence of the geometrical aspect ratio of the cavity on the oscillation peak frequency for series 3 . Plain lines are the natural modes [Eq. (1)]; the red symbol is a specific configuration at the $m_{03}-m_{04}$ modes transition. (in the range $0.55<\mathrm{Fr}<0.85$ ) has no impact on the selected oscillation mode of the cavity, but suggest that the geometrical aspect ratio of the cavity governs the mode selection, as discussed in Sec. III C.

Additionally, Fig. 9 plots the evolution of the oscillation amplitude $\eta_{1 / 3}$ at the downstream edge of the cavity, as a function of the Froude number for series $2 b$ and $2 c$. It appears that while the oscillation mode remains similar, the oscillation amplitude rapidly increases with the Froude number. These results confirm the previous observations by Wolfinger et al. (2012) and Tuna et al. (2013). Series $2 \mathrm{a}$ in the square cavity is not included in Fig. 9 since the seiche behavior is more complex and a single amplitude cannot be representative of the surface oscillations.

\section{Influence of the geometrical aspect ratio}

To assess the influence of the geometrical aspect ratio of the cavity on the selected oscillation mode and peak frequency, 23 flow configurations are considered in series 3 by regularly increasing the width of the cavity while keeping a constant water depth and Froude number. Figure 10 shows the measured peak frequency $f$ in function of the dimensionless cavity width, in which $f$ is made dimensionless

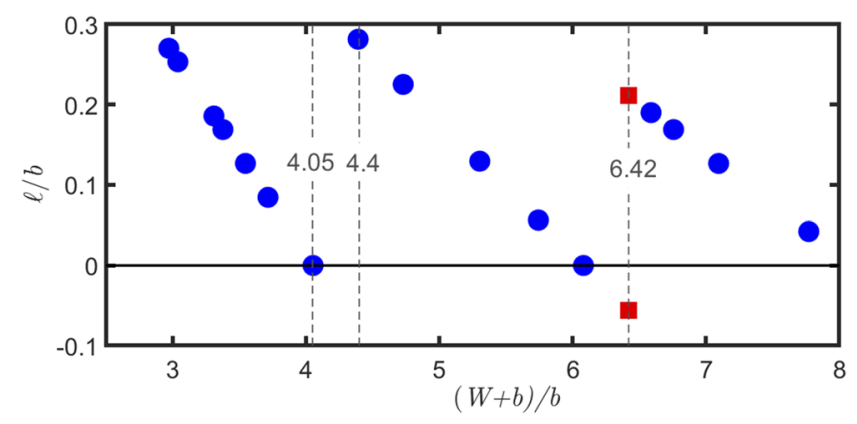

FIG. 11. Evolution of the normalized distance $\ell / \mathrm{b}$ between the interface and the first transverse oscillation node as a function of the aspect ratio of the cavity.

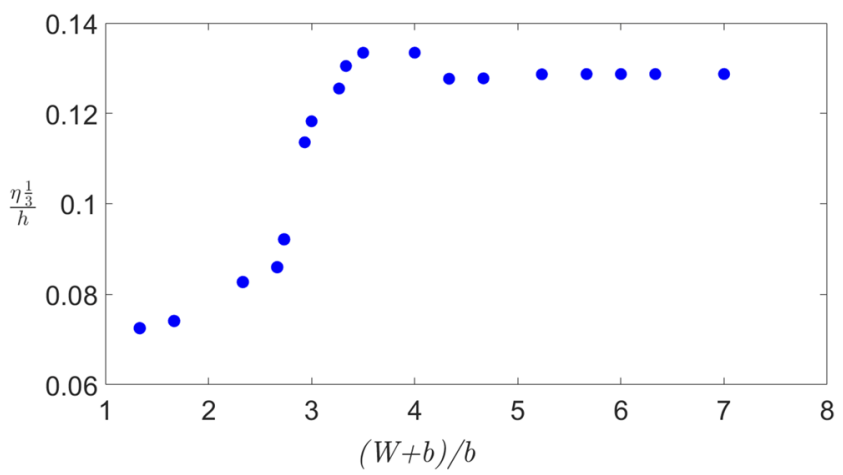

FIG. 12. Evolution of the normalized oscillation amplitude at the downstream edge of the cavity with the aspect ratio of the cavity for series 3 . The authors wish to remind that $\eta_{1 / 3}$ quantifies the amplitude of surface oscillation at the downstream edge of the cavity, which is not necessarily an antinode of the oscillation. The amplitude of the standing wave should be systematically measured at the antinode. 


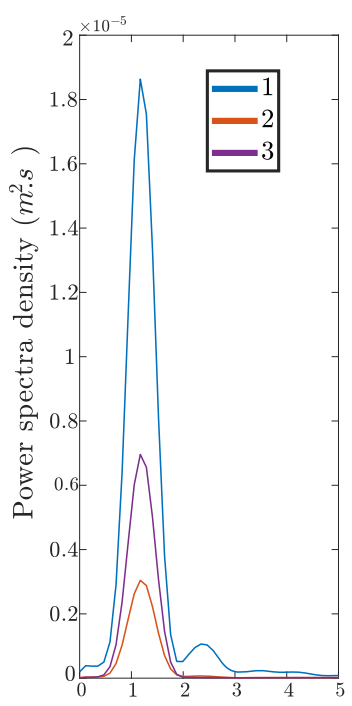

Frequency $(\mathrm{Hz})$

\section{$t / T=0.25$}
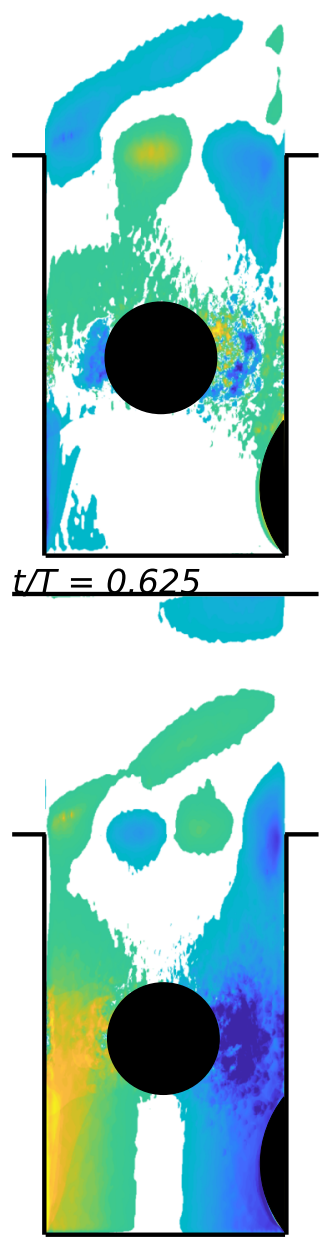

$t / T=0$

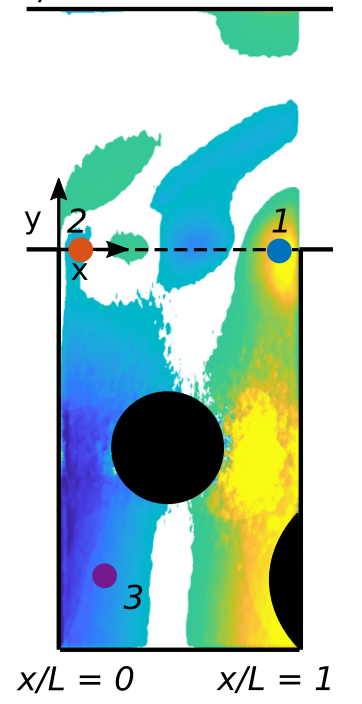

$t / T=0.375$
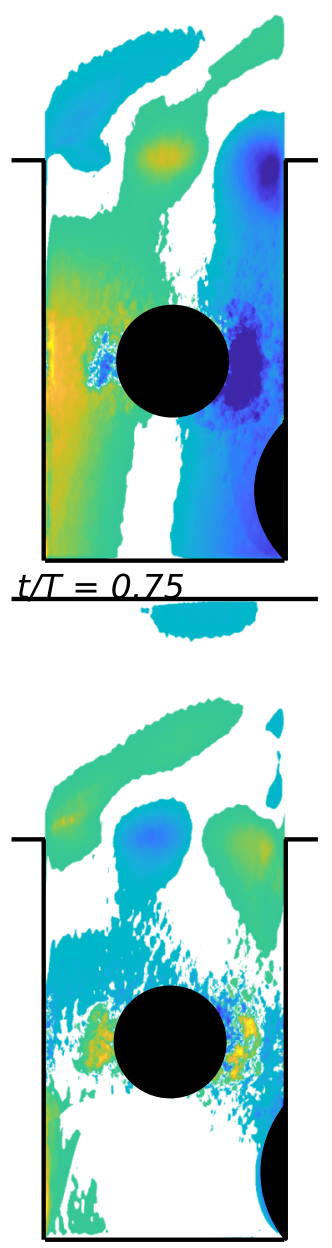

$\frac{t / T=0.125}{\pi} \quad y / L=1$

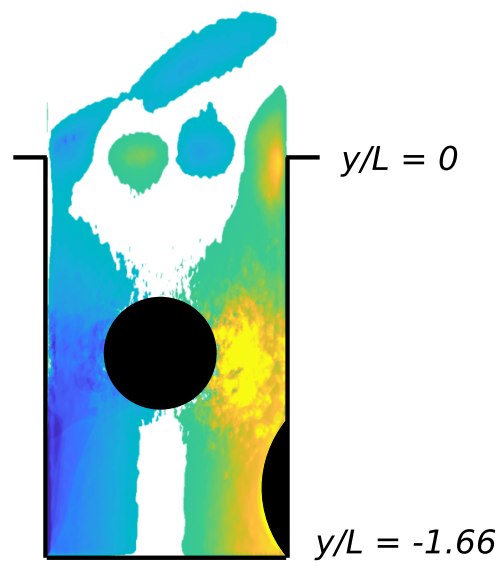

$t \longdiv { T } = 0 . 5$
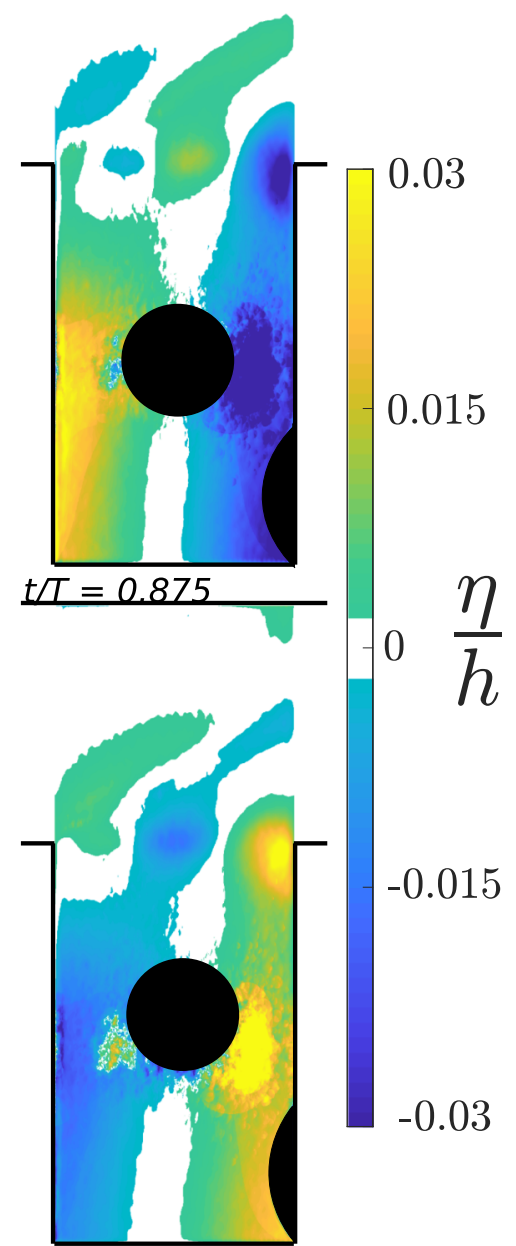

FIG. 13. Time-evolution along one oscillation period of the free-surface deformation (projected on the peak frequency) in the main stream and the cavity for oscillation mode $m_{10}\left(f=f_{10}\right)$ with $[W+b] / b$ $=2.66$ from series 3 . The black region corresponds to the area with direct reflection of light toward the camera, where data are not reliable. The first graph on the top-left depicts the power spectra of the surface registrations at three locations indicated in the second graph on the top row. 
with the natural frequency of the first longitudinal mode $f_{10}$ [as proposed by Wolfinger et al. (2012), Tuna et al. (2013), and Perrot-Minot et al. (2020)]. In such a graph, the longitudinal natural frequency $f_{10}$ appears as a horizontal line while transverse natural frequencies $f_{01}, f_{02}, f_{03}, \ldots$ appear as decreasing curves. Figure 10 confirms that the oscillation mode is strongly dependent on the geometrical aspect ratio of the cavity. Four groups of measurement data with the same oscillation mode arise, corresponding to four ranges of geometrical aspect ratios of cavities, and are detailed below.

For relatively narrow cavities with $[W+b] / b<3$, the uninodal longitudinal mode $m_{10}$ is excited (Fig. 3), in agreement with the oscillation modes reported by Kimura and Hosoda (1997), Wolfinger et al. (2012), Tuna et al. (2013), and Perrot-Minot et al. (2020).

As the dimensionless width of the cavity plus the main stream $[W+b] / b$ exceeds 3 , the oscillation mode becomes transverse and equals to $m_{02}$, which is characterized by two nodes across the main stream and cavity width: one node line being located in the main stream and the other one in the cavity (Fig. 3). The switch in oscillation direction from longitudinal to transverse at $[W+b] / b \sim 3$ is very sudden and robust, i.e., verification of this switch was repeated several times with a high level of reproducibility. Such change of mode from longitudinal to transverse as the width of the cavity increases was already reported by Rabinovich (2009) in bays and harbors. The $m_{02}$ mode $\left(f=f_{02}\right)$ is then observed for cavities with an aspect ratio up to $[W+b] / b \sim 4.05$.

As the aspect ratio $[W+b] / b$ exceeds 4.4 , the oscillation mode switches again, to another transverse mode $m_{03}\left(f=f_{03}\right)$ corresponding with the occurrence of a third node line. In such a case, two nodes are located in the cavity and one in the main stream. This $m_{03}$ mode is systematically observed for $4.4<[W+b] / b<6.42$.

Finally, at $[W+b] / b \sim 6.42$, the oscillation mode switches to transverse mode $m_{04}$ with four node lines, three being located in the cavity and one in the main stream (Fig. 3). This mode is observed herein for the widest cavities up to $[W+b] / b \sim 8$. A specific effort was devoted to precisely locate the transition from mode $m_{03}$ to $m_{04}$. For $[W+b] / b=6.42$, repetitions of the flow configuration exhibit either mode $m_{03}$ or mode $m_{04}$, corresponding to a bifurcation between both modes. This "double" configuration is indicated as a red symbol in Fig. 10.

It is interesting to note that for all configurations with $[W+b] / b$ $>3$, the peak frequency remains comprised in a relatively narrow range: $0.45<f / f_{10}<0.7$, i.e., $0.6 \mathrm{~Hz}<f<0.9 \mathrm{~Hz}$.

Furthermore, Fig. 10 indicates that transitions to higher transverse modes $\left(m_{02}\right.$ to $m_{03}$ and then $\left.m_{04} \ldots\right)$ occur at proportional aspect ratios: $[W+b] / b=4.05-4.4,[W+b] / b=6.42$, etc. Noting $\ell$ the distance along the $y$ axis between the cavity/main stream interface $(y=0)$ and the first node of the transverse modes located the closest to the channel bank (Fig. 2), Fig. 11 plots the evolution of $\ell / b$ as a function of the normalized aspect ratio of the cavity $[W+$ $b] / b$. $\ell$ appears to remain positive for all flow configurations, i.e., the first node always occurs within the main stream area. For $[W$ $+b] / b$ slightly exceeding a transition value $([W+b] / b=4.05-4.4$ or $6.42, \ldots)$, the first node is located relatively far from the interface (within the main stream). As $W$ increases, this node approaches the interface ( $\ell$ decreases) and reaches the interface for $[W+b] / b$ equal to the next transition value ( $\ell$ becomes negative) where the following switch occurs so that the first node moves back into the main stream.

Furthermore, Fig. 12 plots the amplitude of the water surface oscillation at the downstream edge of the cavity for series 3 . It reveals that for narrow cavities $(W+b) / b<3$ with a longitudinal oscillation mode the amplitude remains limited and rapidly increases after the transition to the transverse modes for $(W+b) / b>3$.

\section{RESULTS: SYNCHRONIZATION OF VORTEX DYNAMICS AND SURFACE OSCILLATION}

The fringe projection profilometry gives access not only to the main oscillation mode but also to the trajectories of the vortices shed at the upstream edge of the cavity and impinging at the downstream edge. Indeed, in Figs. 13 and 15, the vortices are recognizable as local depressions of the surface elevation due to centrifugal forces, as previously discussed by Kimura and Hosoda (1997) and Ali et al. (2011). The interactions between the surface oscillation and the vortex trajectories are then analyzed by visualizing in Figs. 13 and 15 the evolution of the fluctuation of surface elevation $\eta$ during one oscillation period $T=1 / f$. Figure 13 depicts a longitudinal mode and Fig. 15 a transverse mode.

\section{A. Longitudinal mode}

Figure 13 plots the fluctuation of the surface elevation every $1 / 8$ of a period for the configuration from series 3 with $[W+b] / b=2.66$. The surface oscillation spectrum in the top-left graph confirms that the oscillation comprises a single peak frequency that is observed at every point of the studied domain. The oscillation mode $m_{10}$ of the seiche is easily recognizable with antinode lines along the upstream and downstream walls of the cavity and a node line along the centerline of the cavity $(x / L=0.5)$, from the cavity/main stream interface to the end wall.

Note, however, that the shape of the surface oscillation in the cavity is slightly deformed compared to the canonical $m_{10}$ mode presented in Fig. 3 (for instance, the node line does not remain perfectly parallel to the cavity walls) due to the combined effect of the main

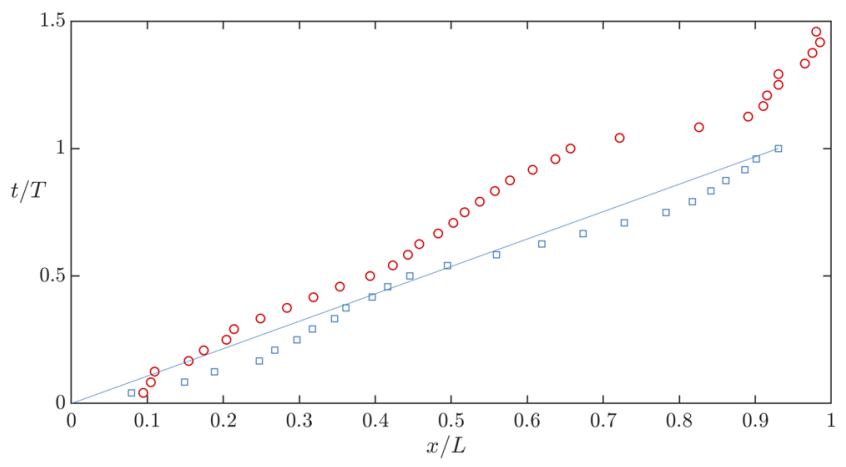

FIG. 14. Trajectory of the vortices along the interface between the cavity and the mainstream for configurations with $[W+b] / b=2.66$ (red circles, mode $m_{10}$ from Fig. 13) and $[W+b] / b=3.33$ (blue squares, mode $m_{02}$ from Fig. 15) from series 3 with a linear trend as plain line. 

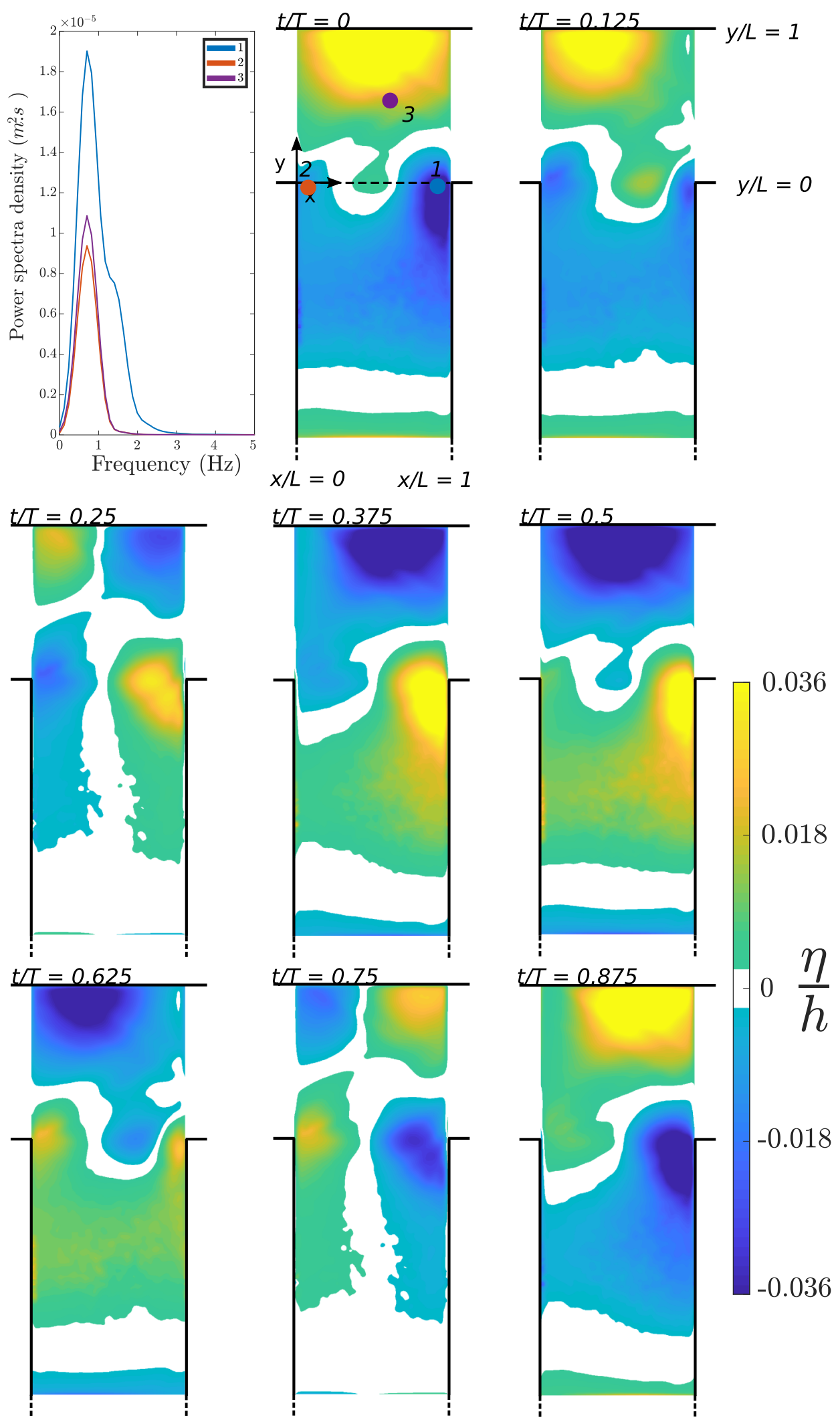

FIG. 15. Same as Fig. 13, but for the $m_{02}$ $\left(f=f_{02}\right)$ oscillation mode with $[W+b] / b$ $=3.33$ from series 3 (part of the cavity is outside the measurement domain). 
stream, mixing layer, and cavity edges. Moreover, a complex oscillation pattern arises within the channel, which will require further investigation for a complete comprehension.

The reference time $t / T=0$ is selected so that a maximum trough (blue color) and a maximum crest (yellow color) occur along, respectively, the upstream and downstream cavity walls. As expected, the opposite takes place at $t / T=0.5$, in agreement with the $m_{10}$ mode and a quite flat free-surface is measured at $t / T=0.25$ and $t / T=0.75$.

At $t / T=0$, a vortex (indicated as a blue patch traveling along the interface) is created at the upstream edge and then travels downstream along the main stream/cavity interface $(y=0)$. Figure 14 plots the evolution along the time of the streamwise location of this vortex. At mid-period $(t / T=0.5)$, the core of the vortex reaches $x / L$ $=0.4$ and continues traveling downstream. At the end of the period $(t / T=1)$, the vortex is located at $x / L=0.66$ and a new vortex is created at the upstream edge, so that two consecutive vortices co-exist along the interface. The first vortex continues moving downstream and impinges the downstream edge at $t / T \sim 1.5$ (i.e., $t / T=0.5$ on Fig. 13). A single vortex thus exists for the second half period of oscillation $(0.5<t / T<1$ or $1.5<t / T<2)$. Globally, the vortex celerity is too small to allow the vortex traveling the whole cavity length in one wave period. Moreover, Fig. 14 (red circles) shows that the vortex celerity evolves within one period. To conclude, the periods of vortex shedding and impinging equal that of the seiche, but the wavelength of the vortex street is $\lambda_{\mathrm{v}}=2 L / 3=\lambda / 3$, with $\lambda$ the wavelength of the seiche $(\lambda=2 L)$.

A major finding is, thus, that the vortices appear at the upstream edge $(t / T=0)$ and impinge the downstream edge $(t / T$ $=0.5$ or 1.5 ) exactly as the maximum trough of the standing wave occurs along the corresponding wall. This co-existence of a trough of the seiche and a depression of the surface elevation inside the vortex seems to be the favorable condition for the appearance/disappearance of the vortices. This behavior is observed on all measured longitudinal oscillation modes and is discussed in Sec. V.

\section{B. Transverse mode}

In a similar way as in Sec. IV A, Fig. 15 shows the timeevolution of surface deformation during an oscillation period, corresponding to the transverse mode $m_{02}$ with $[W+b] / b=3.33$ from series 3. Again, the top-left graph confirms that the oscillation comprises a single peak frequency identically observed all over the studied domain. Unfortunately, the whole cavity width could not be captured at once and only $2 / 3$ of the cavity width is visible in Fig. 15.

At $t / T=0$, the $m_{02}$ node is easily recognizable with a maximum crest (yellow zone) along the channel bank and the end wall of the cavity (not visible here), a maximum trough (blue zone) at the entrance of the cavity and two longitudinal node lines as white areas. As for Fig. 13, the opposite takes place at $t / T=0.5$ and a quite flat surface is measured at $t / T=0.25$ ad 0.75 .

Again, the vortex shedding and impingement, respectively, at the upstream and downstream edges of the interface are both in phase with the free-surface oscillation. At $t / T=0$, a vortex is generated at the upstream edge (see Fig. 14). At $t / T=0.5$, the vortex is located near the center of the mixing layer $(x / L=1 / 2)$. At $t / T=1$, the vortex finally impinges the downstream edge. Consequently, a single vortex exists in the cavity/main stream interface at all times, the vortex shedding period equals that of the seiche, and the wave length of the vortex street equals the cavity length, i.e., half the wave length of the seiche $\lambda_{v}=L=\lambda / 2$. Note that in this case, the vortex celerity is almost constant (see Fig. 14, blue squares) and not affected by the transverse waves. It is also worth noting that this behavior is not only retrieved for all $m_{02}$ modes but also for all $m_{03}$ and $m_{04}$ transverse modes. Besides, Fig. 15 reveals that, for these transverse oscillation modes, a vortex appears at and impinges, respectively, the upstream and downstream edges of the cavity at the time when a maximum trough is located along the mixing layer, i.e., including the upstream and downstream edge of the cavity.

To summarize, Figs. 13 and 15 reveal that for both longitudinal and transverse oscillation modes (i) the vortex shedding is in phase with the surface oscillation (as previously discussed by several authors) and (ii) the vortex generation and impingement always occur in phase with a maximum trough of the seiche, respectively, at the upstream and downstream cavity edge. Consequently, the vortex generation and impingement are in phase with each other for the transverse modes but out of phase for the longitudinal mode.

\section{DISCUSSIONS AND CONCLUSIONS}

The present work aimed at investigating the characteristics of the free-surface oscillation of rectangular lateral cavities (with a fixed length $L$ and variable width $W$ ) adjacent to prismatic main streams of the rectangular cross-section (of specific width $b=L$ ) with a steady 1D flow. This work was dedicated to flows with a high, subcritical, Froude number, comprised between 0.55 and 0.68 . Such free-surface oscillations, often referred to as "seiches," were previously measured in the literature. However, no consensus existed regarding the selection of the oscillation mode (direction of oscillation and number of nodes) so that the first objective of the present work was to establish the impact of the non-dimensional governing parameters on the oscillation mode. Moreover, while most authors in the literature suggested that the impingement at the downstream edge of the vortices traveling along the mixing layer was responsible for the seiching process, no clear understanding of the interactions between the oscillation of the free-surface and the vortex shedding taking place along the cavity/main stream interface was established. The second objective of the present work was then to highlight the synchronization of the vortex dynamics along the mixing layer and the surface oscillation.

The first general result is that for all tested configurations, the oscillation takes the form of a natural cavity mode, oscillating at a frequency equal to a natural frequency of the cavity. The observed modes are one-dimensional, longitudinal or transverse, modes for the rectangular cavities $(W \neq L)$ and a combination of a longitudinal and a transverse mode for the square cavity ( $W=L$, hence with $b$ $+W=2 L)$ (Engelen et al., 2020).

Moreover, we confirm a previous statement that the dimensionless water depth and Froude number of the main stream do not affect the selected oscillation mode. Oppositely, the geometrical aspect ratio of the cavity governs the mode selection with, for an increasing cavity width, a longitudinal mode $m_{10}$ followed by a $m_{02}$, a $m_{03}$, and a $m_{04}$ transverse mode. The transition from longitudinal 
to transverse mode takes place for $[W+b] / b=3$ and the transitions from $m_{02}$ to $m_{03}$ and to $m_{04}$ take place regularly as the aspect ratio [ $W$ $+b] / b$ reaches values about $4.2,6.4$, etc., as the first oscillation node overtakes the cavity/main stream interface $(\ell$ becomes negative, see Fig. 11).

Finally, we confirm the statement from the literature that the seiche and the vortex shedding processes exhibit the same frequency. We also show that these two processes are highly synchronized. Notably, vortices are systematically shed at the upstream edge and impinge the downstream edge of the cavity when a maximum trough of the seiche is located at the corresponding edge.

The last section exposes hypothetical explanations for the synchronizations between the vortex dynamics and seiching. On the one hand, at the downstream edge, the synchronization between the vortex impingement (with a local depression) and a trough of the seiche (with a large scale depression along the downstream wall for the longitudinal mode as at $t / T=0.5$ in Fig. 13 and along the mixing layer for the transverse modes as at $t / T=0$ in Fig. 15) is explained by considering that the vortex impinging the downstream edge is the process responsible for the seiching. The free-surface depression within the vortex reaching the downstream edge transfers oscillation energy to the seiching by "pulling down" the free-surface periodically. On the other hand, at the upstream edge, reasons for the synchronization differ for the longitudinal and transverse modes. First, for a transverse mode, Fig. 15 reveals that the vortex is generated at the upstream edge (at $t / T=0$ ) as a crest occurs along the channel bank and a trough near the mixing layer (including the upstream edge). At this time, the transverse velocity caused by the seiche changes direction and becomes directed from the main stream toward the cavity (along $-y$ ), in agreement with the sense of rotation of the vortices in the mixing layer [see for instance Loucks and Wallace (2012)]. Second, for a longitudinal mode, Perrot-Minot et al. (2020) showed that the vortex shedding frequency is governed by the so-called Rossiter model (adapted to open-channel flows by the authors), and that for an approaching flow with a Froude number of about 0.6 (as in the present work), the solution of the Rossiter model corresponds to longitudinal mode $m_{10}$. The Rossiter model implies that the vortex dynamics and surface oscillations are in phase at the upstream and downstream cavity edges.

However, no explanation could be found to explain neither the transition from the longitudinal to the transverse mode as the geometrical aspect ratio of the cavity exceeds 3 , nor the transition from one transverse mode to another. Understanding these transitions will require further experiments and analytical developments. Moreover, it should be reminded that the present work considered a specific geometrical constraint with an equal value for the channel width and cavity length $L=b$. Future laboratory work should identify how the transitions between the different oscillation modes are affected by a modification of this geometry, particularly with $b \gg$ $L$. Finally, future works in laboratory or field conditions should be dedicated to understand and predict the surface oscillation modes in lateral cavities with more complex and realistic shapes, for which the natural modes and natural frequencies must be computed first.

\section{ACKNOWLEDGMENTS}

This work was performed within the framework of the EUR H2O'Lyon (Grant No. ANR-17-EURE-0018) of Université de Lyon
(UdL), within the program "Investissements d'Avenir" operated by the French National Research Agency (ANR).

\section{APPENDIX A: MEAN STREAMWISE VELOCITY AND TURBULENT INTENSITY IN THE MAIN STREAM UPSTREAM FROM THE CAVITY (FIG. 16)}

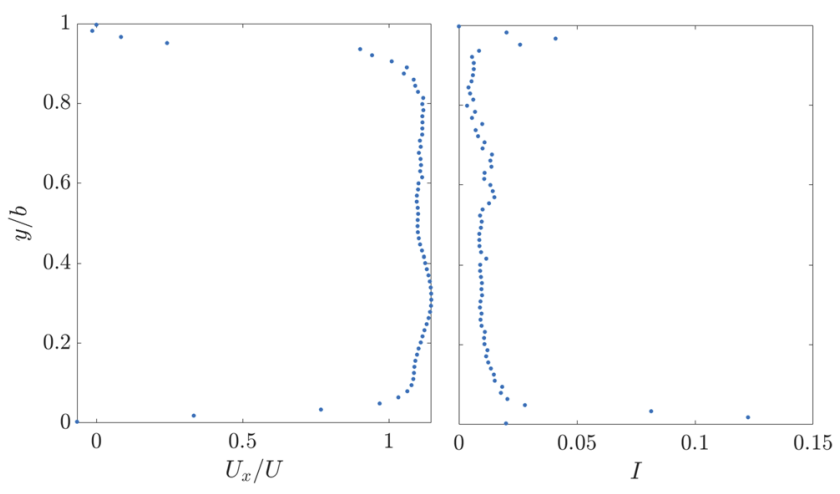

FIG. 16. Transverse profile of mean streamwise velocity $U_{x}$ normalized by the bulk velocity $U$ (left) and turbulent intensity $I=0.5\left(\left\langle u_{x}{ }^{2}\right\rangle+\left\langle u_{y}{ }^{2}\right\rangle\right) / U$ with prime denoting the fluctuant component and \langle\rangle the time averaging (right) in the main stream just upstream from the upstream corner of the cavity, measured by 2D-PIV at elevation $z / h=0.8$ for the configuration from series $2 b$ and 3 with $F r=0.6$ and $(W+b) / b=2.66$.

\section{APPENDIX B: MEASUREMENT REPEATABILITY (FIG. 17)}

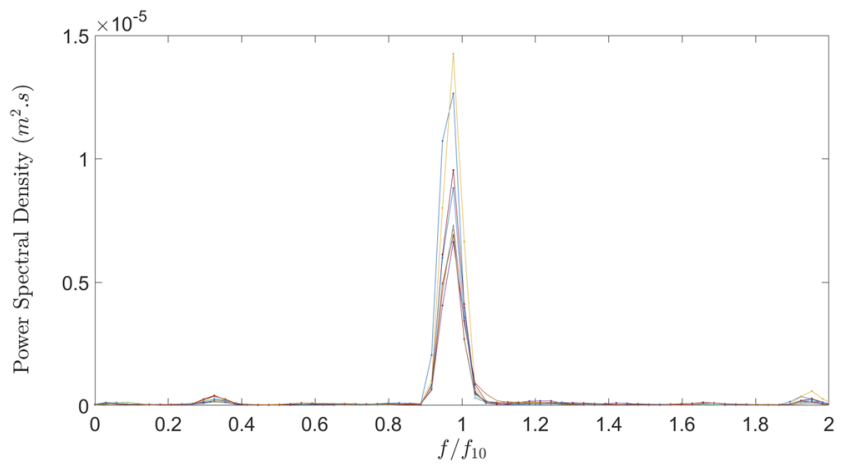

FIG. 17. Spectrum of the water surface oscillation for nine independent repetitions of the same configuration. 


\section{APPENDIX C: ALL MEASUREMENT SERIES AND CORRESPONDING PEAK FREQUENCIES (TABLE III)}

TABLE III. List of flow configurations and measured peak frequencies for each series. Note that for series 1 and $2 \mathrm{a}$ in square cavities, the bi-directional seiching exhibits two peak frequencies.

\begin{tabular}{|c|c|c|c|c|c|c|}
\hline \multirow{2}{*}{ Series } & \multirow{2}{*}{$\begin{array}{c}\mathrm{Fr} \\
0.59\end{array}$} & \multirow{2}{*}{$\begin{array}{c}h / L \\
0.25\end{array}$} & \multirow[t]{2}{*}[W+b]{$/ b$} & \multicolumn{3}{|c|}{ Peak frequency $(\mathrm{Hz})$} \\
\hline & & & & 0.55 & & 1.20 \\
\hline \multirow{5}{*}{ Series 1} & 0.60 & 0.28 & \multirow{5}{*}{2.00} & 0.57 & & 1.22 \\
\hline & 0.61 & 0.30 & & 0.59 & & 1.25 \\
\hline & 0.63 & 0.32 & & 0.60 & & 1.28 \\
\hline & 0.63 & 0.34 & & 0.63 & & 1.30 \\
\hline & 0.63 & 0.38 & & 0.70 & & 1.34 \\
\hline \multirow{19}{*}{ Series $2 \mathrm{a}$} & 0.55 & \multirow{19}{*}{0.36} & \multirow{19}{*}{2.00} & 0.63 & & 1.30 \\
\hline & 0.56 & & & 0.63 & & 1.31 \\
\hline & 0.58 & & & 0.64 & & 1.32 \\
\hline & 0.59 & & & 0.64 & & 1.31 \\
\hline & 0.60 & & & 0.64 & & 1.31 \\
\hline & 0.60 & & & 0.64 & & 1.32 \\
\hline & 0.62 & & & 0.64 & & 1.32 \\
\hline & 0.63 & & & 0.64 & & 1.32 \\
\hline & 0.64 & & & 0.64 & & 1.31 \\
\hline & 0.64 & & & 0.64 & & 1.32 \\
\hline & 0.64 & & & 0.65 & & 1.31 \\
\hline & 0.65 & & & 0.65 & & 1.33 \\
\hline & 0.65 & & & 0.64 & & 1.30 \\
\hline & 0.65 & & & 0.65 & & 1.31 \\
\hline & 0.66 & & & 0.65 & & 1.33 \\
\hline & 0.66 & & & 0.66 & & 1.33 \\
\hline & 0.67 & & & 0.66 & & 1.33 \\
\hline & 0.67 & & & 0.66 & & 1.32 \\
\hline & 0.68 & & & 0.66 & & 1.33 \\
\hline \multirow{4}{*}{ Series $2 b$} & 0.55 & \multirow{4}{*}{0.195} & \multirow{4}{*}{2.66} & \multicolumn{3}{|c|}{1.25} \\
\hline & 0.6 & & & \multicolumn{3}{|c|}{1.22} \\
\hline & 0.62 & & & \multicolumn{3}{|c|}{1.22} \\
\hline & 0.65 & & & \multicolumn{3}{|c|}{1.23} \\
\hline \multirow{3}{*}{ Series $2 c$} & 0.6 & \multirow{3}{*}{0.195} & \multirow{3}{*}{3.00} & \multicolumn{3}{|c|}{0.78} \\
\hline & 0.62 & & & \multicolumn{3}{|c|}{0.77} \\
\hline & 0.65 & & & \multicolumn{3}{|c|}{0.78} \\
\hline & & & 1.33 & & 1.27 & \\
\hline & & & 1.67 & & 1.26 & \\
\hline & & & 2.00 & & 1.27 & \\
\hline & & & 2.33 & & 1.24 & \\
\hline & & & 2.67 & & 1.22 & \\
\hline & & & 2.73 & & 1.22 & \\
\hline & & & 2.93 & & 0.79 & \\
\hline & & & 3.00 & & 0.78 & \\
\hline & & & 3.27 & & 0.73 & \\
\hline & & & 3.33 & & 0.72 & \\
\hline Series 3 & 06 & 0105 & 3.50 & & 0.69 & \\
\hline series 3 & 0.0 & 0.195 & 3.66 & & 0.70 & \\
\hline & & & 4.00 & & 0.61 & \\
\hline
\end{tabular}

TABLE III. (Continued.)

\begin{tabular}{|c|c|c|c|c|}
\hline & & & 4.33 & 0.81 \\
\hline & & & 4.67 & 0.78 \\
\hline & & & 5.23 & 0.70 \\
\hline & & & 5.67 & 0.65 \\
\hline \multirow[t]{6}{*}{ Series } & Fr & $h / L$ & {$[W+b] / b$} & Peak frequency $(\mathrm{Hz})$ \\
\hline & & & 6.00 & 0.62 \\
\hline & & & 6.50 & 0.76 \\
\hline & & & 6.33 & 0.76 \\
\hline & & & 7.00 & 0.70 \\
\hline & & & 7.66 & 0.68 \\
\hline
\end{tabular}

\section{DATA AVAILABILITY}

The data that support the findings of this study are available from the corresponding author upon reasonable request.

\section{REFERENCES}

Ali, M. S., Mahjabin, T., and Ria, S. J., "Numerical simulation of dead zone flows in an open channel with a side cavity and sudden enlargement," J. Civ. Eng. 39(2), 135-148 (2011).

Ardhuin, F., Devaux, E., and Pineau-Guillou, L., "Observation et prévision des seiches sur la côte atlantique française," in XI Journées Nationales Génie Côtier-Génie Civil, Les Sables d'Olonne (Editions Paralia CFL, 2010) (in French).

Arneborg, L. and Liljebladh, B., "The internal seiches in Gullmar Fjord. Part I: Dynamics,” J. Phys. Oceanogr. 31(9), 2549-2566 (2001).

Aubourg, Q. and Mordant, N., "Investigation of resonances in gravity-capillary wave turbulence," Phys. Rev. Fluids 1(2), 023701 (2016).

Cobelli, P. J., Maurel, A., Pagneux, V., and Petitjeans, P., "Global measurement of water waves by fourier transform profilometry," Exp. Fluids 46, 1037-1047 (2009).

Engelen, L., Perrot-Minot, C., Mignot, E., Riviere, N., and De Mulder, T., "Experimental study of bidirectional seiching in an open-channel, lateral cavity in the time and frequency domain," Phys. Rev. Fluids (unpublished) (2020).

Jackson, T. R., Apte, S. V., Haggerty, R., and Budwig, R., "Flow structure and mean residence times of lateral cavities in open channel flows: Influence of bed roughness and shape," Environ. Fluid Mech. 15, 1069-1100 (2015).

Kimura, I. and Hosoda, T., "Fundamental properties of flows in open channels with dead zone," J. Hydraul. Eng. 123(2), 98-107 (1997).

Lamb, H., Hydrodynamics (Cambridge University Press; Dover, 1945).

Le Coz, J., Michalková, M., Hauet, A., Čomaj, M., Dramais, G., Holubová, K., Piégay, H., and Paquier, A., "Morphodynamics of the exit of a cutoff meander: Experimental findings from field and laboratory studies," Earth Surf. Processes Landforms 35(3), 249-261 (2010).

Loucks, R. B. and Wallace, J. M., "Velocity and velocity gradient based properties of a turbulent plane mixing layer," J. Fluid Mech. 699, 280-319 (2012).

Meile, T., Boillat, J.-L., and Schleiss, A. J., "Water-surface oscillations in channels with axi-symmetric cavities," J. Hydraul. Res. 49(1), 73-81 (2011).

Mignot, E., Cai, W., Launay, G., Riviere, N., and Escauriaza, C., "Coherent turbulent structures at the mixing-interface of a square open-channel lateral cavity," Phys. Fluids 28, 045104 (2016). 
Mignot, E., Cai, W., Polanco, J. I., Escauriaza, C., and Riviere, N., "Measurement of mass exchange processes and coefficients in a simplified open-channel lateral cavity connected to a main stream,” Environ. Fluid Mech. 17, 429-448 (2017).

Mignot, E., Pozet, M., Riviere, N., and Chesne, S., "Bidirectional seiching in a rectangular, open-channel, lateral cavity," in Proceeding of 22nd CFM, Lyon, France, 2015.

Perrot-Minot, C., Mignot, E., Perkins, R., Perkins, D., and Riviere, N., "Vortex shedding frequency in open-channel lateral cavity," J. Fluid Mech. 892, A25 (2020).

Rabinovich, A. B., in Seiches and Harbor Oscillations (World Scientific Publisher, Singapore, 2009), Chap. IX.

Rossiter, J. E., "Wind-tunnel experiments on the flow over rectangular cavities at subsonic and transonic speeds," Technical Report No. 64037 and Aeronautical Research Council Reports and Memoranda No. 3438, 1964.
Sandoval, J., Mignot, E., Mao, L., Pastén, P., Bolster, D., and Escauriaza, C., "Field and numerical investigation of transport mechanisms in a surface storage zone," J. Geophys. Res.: Earth Surf. 124(4), 938-959, https://doi.org/10.1029/2018jf004716 (2019).

Sanjou, M., Okamoto, T., and Nezu, I., "Dissolved oxygen transfer into a square embayment connected to an open-channel flow," Int. J. Heat Mass Transfer 125, 1169-1180 (2018).

Tsubaki, R. and Fujita, I., "Surface oscillations in flow past a side cavity using stereoscopic measurement and POD," J. Hydrosci. Hydraul. Eng. 24(2), 41-51 (2006).

Tuna, B. A., Tinar, E., and Rockwell, D., "Shallow flow past a cavity: Globally coupled oscillations as a function of depth," Exp. Fluids 54, 1586 (2013).

Wolfinger, M., Ozen, C. A., and Rockwell, D., "Shallow flow past a cavity: Coupling with a standing gravity wave," Phys. Fluids 24, 104103 (2012). 